\title{
Trabalho de Conclusão de Curso de graduação em saúde: Dificuldades e estratégias
}

\section{Undergraduate monograph health: Problems and strategies}

\author{
Flavia Mazzoli-Rocha \\ PhD. Centro Universitário Serra dos Órgãos. E-mail: flamazzoli@gmail.com \\ Andrea Serra Graniço \\ MSc. Centro Universitário Serra dos Órgãos. E-mail: coordcursofisioterapia@unifeso.edu.br \\ Alba Barros Souza Fernandes \\ PhD. Centro Universitário Serra dos Órgãos. E-mail: alba.fernandes@gmail.com
}

\begin{abstract}
Resumo: Objetivou-se descrever dificuldades vivenciadas por professores e pesquisadores diante da elaboração do Trabalho de Conclusão de Curso de Graduação na grande área da Saúde. As estratégias para auxiliar o discente universitário a escrever seu Trabalho de Conclusão de Curso com qualidade técnica, foram pontuadas de forma a auxiliar discentes e orientadores, como fornecer: subsídios para facilitar a escrita da monografia, desde a disponibilização de disciplina de Português à Diretrizes Metodológicas; e informações sobre plágio e seus mecanismos de detecção. A disponibilização destas estratégias pode ser o diferencial na elaboração de um Trabalho de Conclusão de Curso de melhor qualidade.
\end{abstract}

Palavras-chave: Monografia; Educação em saúde; Plágio.

Abstract: The objective was to describe difficulties experienced by teachers and researchers before the elaboration of the undergraduate conclusion work in the great area of Health. The strategies to help the university student to write their Course Completion Work with technical quality, were scored in order to help students and counselors, such as: facilitating the writing of the completion work course, from the provision of Portuguese subject to Methodological Guidelines; and information about plagiarism and its detection mechanisms. The availability of these strategies can be the differential in the elaboration of a completion work course of better quality.

Key words: Monograph; Education in health; Plagiarism. 


\section{INTRODUÇÃO}

A escrita científica determina, ao longo dos anos, a progressão da ciência e a disseminação da informação. Entretanto, o poder da escrita nem sempre acompanha a formação e o amadurecimento do ser humano como profissional (REYNOLDS; THOMPSON, 2011). Inclusive, considerando a relevância da boa escrita na formação profissional, é intrigante o fato de o ensino da escrita não estar presente de forma central na educação científica, uma vez que poucos pesquisadores/educadores avaliam a habilidade de seus estudantes para escrever (CHUR-HANSEN; VERNON-ROBERTS, 2000) ou se esforçam em ensiná-los a escrever de forma clara e concisa (CHUR-HANSEN, 2000).

Ressalta-se ainda que não apenas os mecanismos para ensinar-a-escrever devem ser inseridos pelos educadores no meio acadêmico-universitário, mas também as estratégias de escrever-para-aprender (MOSKOVITZ; KELLOGG, 2011), uma vez que a escrita representa uma ferramenta efetiva no aprendizado e na formação continuada (CARTER et al., 2007; REYNOLDS et al., 2012). Adicionalmente, a relevância da leitura deve ser pontuada. Um estudante que pratica a leitura frequentemente possui facilidades na escrita. Afinal, a leitura fornece conhecimento para elaboração do texto, além de contribuir para a percepção do leitor sobre produção (como os textos lidos são produzidos) e estruturas de escrita (como as informações dos textos lidos estão organizadas) (ORLANDI; GUIMARÃES, 1985).

Em território nacional, a presença de distintas formações básicas e níveis de alfabetismo culmina em expressivas dificuldades vividas pelos estudantes universitários para interpretar e construir um texto (INAF, 2011), sendo um lamento constante dos professores e pesquisadores (REYNOLDS; THOMPSON, 2011). No entanto, este problema precisa ser superado para a elaboração do Trabalho de Conclusão de Curso ou Monografia, exigência das Diretrizes Curriculares Nacionais dos cursos de graduação em Fisioterapia (BRASIL, 2002). A incapacidade de escrever de forma autônoma e a praticidade de "copiar-e-colar" textos da internet facilitam a inserção do discente no mundo do plágio (DE VOSS; ROSATI, 2002; BILIC-ZULLE et al., 2005; COLE, 2007; CARVALHO et al., 2014).

Diante do exposto, a presente revisão objetivou descrever as dificuldades presentes ao longo das etapas que envolvem a elaboração do Trabalho de Conclusão de Curso (a falta de domínio de leitura-escrita e o plágio) e possíveis estratégias para as mesmas.

\section{MATERIAL E MÉTODOS}

Trata-se de uma revisão com o objetivo de conhecer a produção científica associada ao tema de estudo, que permitiu a síntese de diferentes estudos publicados e possibilitou conclusões gerais a respeito das dificuldades mais frequentes ao longo da elaboração do Trabalho de Conclusão do Curso de Graduação em Saúde.

A coleta de dados foi realizada nos meses de fevereiro a maio de 2016, utilizando-se os descritores:
Educação em Saúde, Monografia e Plágio, além de suas versões na língua Inglesa: Monograph, Education in Health, Plagiarism. As bases de dados utilizadas foram a SciELO (Scientific Eletronic Library Online) e Medline (US National Library of Medicine).

Visando o direcionamento da busca bibliográfica, utilizou-se a ferramenta filtrar, sendo selecionados apenas estudos: disponíveis online e na íntegra, em idioma Inglês, Espanhol ou Português, publicados de 2000 a 2016. Adicionalmente, utilizou-se Normas e Resoluções da Secretaria de Educação e do Código Penal (para embasar legalmente algumas informações) além de acessos virtuais a sites sobre Plágio (softwares) e do Ministério da Educação.

Uma leitura exploratória e seletiva foi realizada paralelamente à seleção do material bibliográfico. Posteriormente, uma leitura interpretativa permitiu a organização lógica do assunto que subsidiou a construção do texto.

\section{RESULTADOS E DISCUSSÃO}

Uma formação básica adequada deve prover alfabetismo pleno aos indivíduos. No entanto, conforme dados nacionais, nem todos os universitários possuem este nível de aprendizado (INAF, 2011). A dificuldade na leitura de textos longos, por vezes escritos em inglês, e na escrita autônoma gera insegurança e sensação de incapacidade no momento da elaboração do Trabalho de Conclusão de Curso, facilitando a prática de plágio.

\section{Dificuldade: A falta de domínio da escrita}

Divulgado a cada dois anos pelo Instituto Paulo Montenegro (IPM), o Indicador de Alfabetismo Funcional (INAF) disponibiliza o nível de domínio da leitura, da escrita (letramento) e da Matemática (numeramento) da população brasileira em geral. O teste de alfabetismo inclui leitura e interpretação de textos (bilhetes, notícias, instruções, textos narrativos, gráficos, tabelas, mapas, anúncios), além da aplicação de questionário (abordando características sóciodemográficas e práticas de leitura, escrita e cálculo), ambas relacionadas ao cotidiano. Com base nestes testes, o INAF divide a população em quatro níveis de alfabetismo: analfabetismo absoluto, alfabetismo rudimentar, alfabetismo básico e alfabetismo pleno (Quadro 1).

Segundo o INAF 2011, 27\% da população brasileira com idade entre 15 e 64 anos são analfabetos funcionais. Destes, 6\% incluem analfabetos absolutos (incapazes de ler e escrever) e $21 \%$, alfabetizados de nível rudimentar (capazes de ler textos curtos e lidar com números em operações simples). Por outro lado, os alfabetos funcionais compreendem $73 \%$ da população brasileira, sendo $47 \%$ de nível básico e $26 \%$ de nível pleno (INAF, 2011). Vale mencionar que nos últimos 10 anos, o nível básico demonstrou maior crescimento (de $34 \%$ para $47 \%$ ), enquanto o percentual dos que atingem o nível pleno não variou (INAF, 2011). 
Quadro 1. Níveis de alfabetismo, segundo o Indicador de Alfabetismo Funcional (INAF)

\begin{tabular}{|c|c|c|}
\hline Classificação & Níveis & Características \\
\hline \multirow[b]{2}{*}{$\begin{array}{l}\text { Analfabetismo } \\
\text { funcional }\end{array}$} & $\begin{array}{l}\text { Analfabetismo } \\
\text { absoluto }\end{array}$ & $\begin{array}{l}\text { - Incapaz de realizar tarefas simples (leitura de palavras e frases). } \\
\text { - Pode apresentar potencial para ler números familiares (números de } \\
\text { telefone e preços). }\end{array}$ \\
\hline & $\begin{array}{l}\text { Albabetismo de } \\
\text { nível rudimentar }\end{array}$ & $\begin{array}{l}\text { - Localiza informação explícita em textos curtos e familiares (leitura de } \\
\text { anúncio ou pequena carta). } \\
\text { - Le e escreve números usuais, realizando operações simples (manuseio } \\
\text { de fita métrica e de dinheiro para pagamento de pequenas quantias). }\end{array}$ \\
\hline \multirow{2}{*}{$\begin{array}{l}\text { Alfabetismo } \\
\text { funcional }\end{array}$} & $\begin{array}{l}\text { Albabetismo de } \\
\text { nível básico }\end{array}$ & $\begin{array}{l}\text { - Pessoas consideradas funcionalmente alfabetizadas (leitura e } \\
\text { compreensão de textos médios). } \\
\text { - Limitações quando as operações requeridas envolvem maior número de } \\
\text { elementos, etapas ou relações (leitura de números na casa dos milhões, } \\
\text { resolução de problemas envolvendo sequência simples de operações com } \\
\text { noção de proporcionalidade). }\end{array}$ \\
\hline & $\begin{array}{l}\text { Albabetismo de } \\
\text { nível pleno }\end{array}$ & $\begin{array}{l}\text { - Compreende e interpreta textos em situações usuais, sem restrições } \\
\text { (leitura de textos mais longos, analisando e relacionando suas partes, } \\
\text { compara e avalia as informações, distinguindo fato de opinião). } \\
\text { - Resolve problemas que exigem maior planejamento e controle } \\
\text { (realização de cálculo de área, percentuais e proporções, além de } \\
\text { interpretação de tabelas, mapas e gráficos). }\end{array}$ \\
\hline
\end{tabular}

Quando analisamos o INAF por escolaridade nos últimos 10 anos (população brasileira entre 15 e 64 anos), observamos que algum avanço ocorreu nos níveis de alfabetismo do ensino fundamental I (queda de $8 \%$ de analfabetos funcionais e aumento de $10 \%$ do nível básico). No entanto, nota-se uma queda do nível pleno do ensino fundamental II $(22 \%$ para $15 \%)$ e do ensino médio $(49 \%$ para 35\%). Os dados demonstram que apesar de significativa ampliação da proporção de pessoas que alcança o ensino médio, há uma forte redução no nível de habilidades que aquele nível de ensino consegue garantir para a maioria dos estudantes. A redução das habilidades adquiridas na escolaridade básica reflete no nível superior (enquanto o percentual de brasileiros que alcança a universidade aumenta, o desempenho médio do grupo reduz), onde a proporção de alfabetizados em nível pleno reduziu 14 pontos percentuais (de $76 \%$ para $62 \%$ ) de 2001 para 2011.

Vale mencionar que, segundo as Diretrizes Curriculares Nacionais da Educação Nacional, o ensino fundamental deve objetivar a formação básica do estudante, apresentando como meios básicos o pleno domínio da leitura, da escrita e do cálculo (DIRETRIZES CURRICULARES NACIONAIS DA EDUCAÇÃO NACIONAL, 2013), ou seja, o estudante deve apresentar nível pleno de alfabetismo para ingressar no ensino médio (INAF, 2011). Portanto, o nível pleno de alfabetismo, dotado de capacidade para leitura de textos longos, elaboração de redação e realização de análises críticas, deveria ser indispensável para o ingresso ao ensino superior, embora os dados do INAF mostrem uma realidade diferente.

Esta problemática recai sobre os docentes universitários, que além de orientar seus estudantes, precisam ensiná-los a ler e escrever textos mais complexos. Adicionalmente, as Diretrizes Curriculares Nacionais (BRASIL, 2002) exigem a apresentação de um Trabalho de Conclusão de Curso (TCC), que por sua vez necessita do nível pleno de alfabetismo para ser adequadamente elaborado. Para possibilitar a alta exigência (apresentação de TCC), apesar da reduzida capacidade de ler e escrever (pequeno percentual de nível pleno no nível superior), faz-se necessária a implantação de disciplinas/módulos de Metodologia de Pesquisa e a atuação insistente do Orientador na transformação de seu orientando em um pesquisador e profissional independente.

De acordo com Reynolds e Thompson (2011), dois modelos comuns podem ser utilizados pelos professores para auxiliar a escrita de trabalhos de graduação. $\mathrm{O}$ primeiro trata-se de uma supervisão individualizada, tendo como ponto positivo a garantia da atenção personalizada e como ponto negativo a dedicação excessiva do professor que, por vezes, reescreve o texto para o estudante. $\mathrm{O}$ segundo oferece um curso assistencial para elaborações textuais, no qual o estudante aprende a natureza estrutural e algumas convenções envolvidas no processo de escrever. No entanto, departamentos de ciências apresentam dificuldades relacionadas ao tempo e à falta de recursos humanos especializados em pedagogia, complicando a atuação ativa do docente no processo. Segundo Rocha et al. (2010), além da carga horária do discente destinada à elaboração do TCC, também é preciso adequar o corpo docente para a orientação, capacitando-os e facilitando a formação de uma boa relação orientador-orientando.

No meio universitário, coordenadores e professores esperam que seus estudantes se capacitem técnica e teoricamente, refletindo de forma crítica sobre sua profissão, a partir da leitura e compreensão dos autores da sua área. Desta forma, estes estudantes universitários estarão aptos a elaborar o TCC de forma contextualizada e sem a prática de plágio.

\section{Dificuldade: $O$ plágio}

Apresentar como próprio o trabalho ou a obra intelectual de outra pessoa, de forma integral ou parcial, configura plágio. Falando especificamente do plágio no 
meio acadêmico, este ocorre quando um estudante retira ideias, conceitos ou frases de outro autor (que as formulou e as publicou em arquivos impressos ou digitais), sem citá-lo como fonte de pesquisa (MARTIN, 1992; BILICZULLE et al., 2005). O plágio acadêmico pode resultar de diferentes fatores, incluindo ignorância sobre o assunto e sobre as consequências legais para quem o pratica, oportunidade diante da incapacidade de escrita, tecnologia, valores éticos e competição (HARRIS, 2001; BILIC-ZULLE et al., 2008).

Apesar da prática de plágio ter implicações cíveis e penais e do meio universitário se fundamentar pela criação e pelo desenvolvimento do conhecimento, o plágio pode ser facilmente encontrado em trabalhos acadêmicos (HRABAK et al., 2004; BILIC-ZULLE et al., 2008; KIM et al., 2016). Atualmente no Brasil, o plágio é enquadrado juridicamente na Lei Federal no 9.610/1998, revogada pela Lei Federal n ${ }^{\circ} 12.853 / 2013$, que trata dos direitos autorais e considera contrafação a reprodução não autorizada de uma obra, estando os infratores sujeitos às sanções civis e penais cabíveis (BRASIL, 1998). Adicionalmente, o plágio é previsto no artigo 184 do Código Penal, que trata dos crimes contra a propriedade intelectual e prevê pena com detenção de três meses a um ano ou multa (BRASIL, 1940).

Preocupantemente, a reprodução de palavras e ideias de outros autores como se fossem próprias tende a tornar-se mais frequente, em virtude da facilidade de acesso à informação. Afinal, com um computador e a internet como fonte de informação, é possível "copiar-ecolar" qualquer texto com apenas alguns cliques (DE VOSS; ROSATI, 2002; BILIC-ZULLE et al., 2005; COLE, 2007;; CARVALHO et al., 2014). Inicialmente, trabalhos acadêmicos plagiados poderiam ser facilmente identificados por professores que conhecem o perfil, o estilo de escrita e o nível de conhecimento dos seus estudantes. No entanto, a prática de plágio pelos estudantes vem sendo aprimorada e uma rápida pesquisa em um buscador eletrônico (como Google) pode não ser suficiente para identificá-lo (WEEK, 2006; STANLICK, 2008). Adicionalmente, Bilic-Zulle et al. (2008) observaram que a prática do plágio acontece mesmo após instrução da contrafação, justamente em virtude da difícil detecção da mesma. Atualmente, recomenda-se a utilização de ferramentas eletrônicas de detecção do plágio (softwares), que seguem como bons aliados neste combate (BILIC-ZULLE et al., 2005; BILIC-ZULLE et al., 2008; STANLICK, 2008).

Entretanto, cabe argumentar que é mais importante evitar o plágio do que reconhecê-lo e puni-lo, embora isso seja imprescindível para que a prática não se torne banal. Estudos indicam falta de conhecimento de profissionais e estudantes universitários sobre questões éticas (SHIRAZI et al., 2010; GHAJARZADEH et al., 2012; GHAJARZADEH et al., 2013), demonstrando que a informação pode ser uma aliada fundamental na prevenção. Adicionalmente, existe o interesse de ambos (professores e estudantes) em adotar medidas para combater o plágio (BADEA-VOICULESCU, 2013; RATHORI et al., 2015), que podem ser mensuradas através de questionário (MAVRINAC et al., 2010; GHAJARZADEH et al., 2012; GHAJARZADEH et al.,
2013). Esta questão exige reflexão e posicionamento da Instituição e dos orientadores envolvidos.

\section{Estratégias para a produção de trabalho de conclusão de curso}

Os cursos de graduação, especialmente na Área da Saúde, podem disponibilizar em seu currículo não apenas conceitos de pesquisa e a inicialização antecipada do TCC (disciplina sobre Diretrizes Metodológicas), mas também mecanismos de ensinar-a-escrever e escreverpara-aprender (disciplina de Português).

Em relação às Diretrizes Metodológicas, esta disciplina deve ser introduzida dois anos antes da finalização do curso, permitindo que o discente realize a elaboração do Projeto de TCC. Nesta disciplina, deve haver boa relação entre o professor responsável pela mesma e a equipe de Orientadores, visando garantir a elaboração de um TCC de qualidade, como previsto pelas Diretrizes Curriculares Nacionais (CNE/CES, 2002).

O discente deve ter autonomia para optar por elaborar um Projeto de Pesquisa Científica ou de Revisão Bibliográfica, de acordo com sua disponibilidade e interesse, devendo respeitar o cronograma da disciplina. Algumas etapas devem ser inicialmente propostas para facilitar a construção inicial do Projeto de TCC, como escolha da Área Temática de Estudo (de acordo com as Áreas de Estudo do Curso de Graduação) e escolha do Orientador. Desta forma, o discente será capaz de definir o Tema de interesse relacionado à área pretendida (BEUREN et al., 2003), que pode ser feito em conjunto com seu Orientador.

O estudante então precisa definir a Delimitação do Tema, influenciada pela motivação e curiosidade do estudante em pesquisar um assunto específico de forma aprofundada, bem como pela leitura de arquivos e participação em eventos científicos vinculados à área (BEUREN et al., 2003). Neste momento, entendemos que o Orientador pode ser um grande aliado, não apenas pela inexperiência do estudante, mas também diante da Delimitação de um tema além das capacidades do próprio discente (por tempo ou por estrutura).

Em seguida, é preciso formular a Problemática (questionamentos sobre o assunto escolhido, expresso em forma de pergunta) e possível Hipótese para a mesma (afirmação categórica que tenta responder o Problema levantado, podendo ser confirmada ou negada com o resultado da pesquisa) (BEUREN et al., 2003) e definir os Objetivos (metas), determinando onde o pesquisador quer chegar com a realização do trabalho de pesquisa. Podem ser divididos em Objetivo Geral (único e inspirador para o título do trabalho) e Objetivos Específicos (relacionados aos subproblemas que compõem o Objetivo Geral) (BEUREN et al., 2003).

A elaboração de Introdução e Justificativa deve ocorrer de acordo com o conhecimento adquirido sobre o Problema. Comumente, três a quatro parágrafos são suficientes para embasar o Tema proposto na Introdução do Projeto de Pesquisa, que deve conter informações gerais do assunto, além do Problema e da Hipótese previstos inicialmente (CALS; KOTZ, 2013a; SENGUPTA, 2014). Na Justificativa, é preciso convencer que o trabalho de pesquisa é fundamental de ser efetivado, exaltando a importância do Tema a ser estudado. 
A Metodologia de Pesquisa Científica deve ser elaborada de forma a conter informações minuciosas e detalhadas, permitindo que outro pesquisador reproduza a pesquisa. As informações necessárias incluem desenho do estudo, critérios de elegibilidade, grupos experimentais, coleta de dados (instrumento utilizado), análise de dados e aprovação ética (KOTZ; CALS, 2013; SENGUPTA, 2014). Para a Metodologia de Revisão Científica, deve ser realizada descrição do desenho do estudo, da coleta de dados (ferramentas de busca de referências como uso de bancos de dados específicos) e análise dos dados (como foi a escolha dos artigos a serem utilizados).

Por fim, o Cronograma da pesquisa (previsão de tempo para realização das atividades), as Referências (item obrigatório que deve conter os documentos e qualquer fonte de informação para a elaboração do Projeto) (CALS; KOTZ, 2013b) e os Anexos (documento esclarecedor incluído caso haja necessidade) devem ser organizados.

O início antecipado da elaboração do Projeto de TCC (ao longo do $3^{\circ}$ ano da Graduação) facilita a posterior construção do TCC, uma vez que estudante e Orientador dispõem de mais tempo para a produção do material acadêmico.

A transformação do Projeto de TCC em TCC exige uma construção do Desenvolvimento Teórico através de revisão bibliográfica. O estudante deve realizar um levantamento bibliográfico que dê suporte à fundamentação teórico-metodológica para solucionar o Problema levantado (BEUREN et al., 2003), além da elaboração de Resultados, Discussão e Conclusão. Os achados devem ser apresentados nos Resultados de forma clara, sucinta e objetiva (sem interpretação), de preferência no passado e na ordem da descrição da Metodologia. O termo "diferença significativa" deve ser utilizado para descrever alterações encontradas em termos estatísticos (KOTZ; CALS, 2013). A Discussão deve contextualizar e confrontar os achados principais com os achados de outras pesquisas (CALS; KOTZ, 2013c), enquanto a Conclusão deve finalizar o TCC de forma sucinta, concluindo os achados.

É imprescindível que o estudante crie seu próprio roteiro de leitura e escrita e indicando o tempo a ser destinado para o cumprimento de cada etapa, mas ciente de que modificações acontecerão à medida que o TCC se desenvolve, podendo alterar a programação inicial.

Entretanto, conforme mencionado anteriormente, para a adequada elaboração do TCC, o fornecimento de bases metodológicas não é a única estratégia indispensável. O fornecimento de mecanismos de ensinara-escrever e escrever-para-aprender (disciplina de Português) torna-se fundamental. Em nível internacional, os professores e pesquisadores identificam a necessidade de uma disciplina para melhorar o uso da língua Inglesa, uma vez que muitos de seus estudantes possuem nacionalidade estrangeira (CHUR-HANSEN, 2000; CHUR-HANSEN; VERNON-ROBERTS, 2000). Entretanto, em nível nacional, parece que esta necessidade existe em virtude do nível inadequado de alfabetismo dos estudantes universitários (INAF, 2011). Por razões distintas, a implantação de uma disciplina da língua nacional parece ser uma estratégia crucial na garantia do domínio da escrita e leitura da mesma em ambientes universitários. Vale mencionar ainda a importância da auto-avaliação do estudante em relação a este domínio, possibilitando a identificação de suas fragilidades na leitura e na escrita da língua a ser utilizada (CHURHANSEN, 2000; CHUR-HANSEN; VERNONROBERTS, 2000).

\section{Estratégias visando minimizar o plágio acadêmico}

Os Cursos de Graduação em Saúde devem promover devida informação sobre plágio e ferramentas de auxílio na escrita do TCC conforme sugerido acima, de forma que a prática de plágio seja prevenida. Entretanto, apesar de atuar preventivamente, estas condutas não impedem a prática do plágio. Diante disto, os cursos podem utilizar ferramentas de detecção de plágio, disponíveis online, como: CopySpider, Ephorus, Farejador de plágio, Plagiarism advice, Plagiarism Detect, Turnitin, Viper, entre outros.

Vale ressaltar que os softwares anti-plágio não apontam de fato o plágio. Tratam-se de ferramentas que permitem a identificação de suspeita de plágio através do reconhecimento de informações semelhantes em documentos disponíveis na Internet. Com base na leitura do documento e em algumas configurações básicas do usuário, o software executa pesquisas sequenciais de trechos contínuos do documento em sites de busca. Ao término das buscas, o programa aplica técnicas de processamento de dados a fim de identificar o que é ou não copiado da Internet (PEZZIN, 2010).

Vale mencionar aqui alguns problemas relacionados à pesquisa pelo software. A avaliação do TCC por inteiro gera conflitos de identificação no software (todas as referências, por exemplo, são identificadas como plágio), devendo ser analisado, portanto, apenas os tópicos Introdução, Fundamentação Teórica e Discussão. Adicionalmente, as suspeitas de plágio identificadas pelos softwares se referem apenas à cópia de arquivos online, ou seja, cópias de livro podem não ser identificadas.

Diante destes problemas, uma avaliação qualitativa do TCC deve ser realizada, identificando parágrafos sem referência, parágrafos seguidos com a mesma referência, parágrafos longos com apenas uma referência e parágrafos com referência apenas de livro, que comumente indicam suspeita de plágio. Esta avaliação qualitativa pode ser realizada pelo próprio Orientador ao longo da elaboração do TCC. Desta forma, o estudante pode reescrever os tópicos de forma antecipada e a Instituição terá maior garantia da inexistência de plágio em seus trabalhos.

\section{CONCLUSÕES}

Entende-se que duas estratégias tornam-se fundamentais minimizarmos as fragilidades na elaboração de TCC: o fornecimento de subsídios para facilitar a escrita do TCC, desde a disponibilização de disciplina de Português à Diretrizes Metodológicas; e o fornecimento de informações sobre plágio e mecanismos de detecção do mesmo. 


\section{REFERÊNCIAS}

BADEA-VOICULESCU, O. Attitude of Romanian medicine students towards plagiarism. Rom J Morphol Embryol, v.54, n.3, p.907-908, 2013.

BEUREN, I. M.; LONGARAY, A. A.; RAUPP, F. M.; SOUSA, M. A. B.; COLAUTO, R. D.; PORTON, R. A. B. Como elaborar trabalhos monográficos em Contabilidade - Teoria e Prática. São Paulo: Editora Atlas S.A., 2003.

BILIC-ZULLE, L.; AZMAN, J.; FRKOVIC, V.; PETROVECKI, M. Is there an effective approach to deterring students from plagiarizing? Sci Eng Ethics, v.14, p.139-147, 2008.

BILIC-ZULLE, L.; FRKOVIC, V.; TURK, T.; AZMAN, J.; PETROVECKI, M. Prevalence of plagiarism among medical students. Croat Med J, v.46, n.1, p.126-131, 2005.

BRASIL. Decreto-Lei no 2.848 de 7 de dezembro de 1940 Código penal.

BRASIL. Lei Federal no 9.610 de 19 de fevereiro de 1998. Altera, atualiza e consolida a legislação sobre direitos autorais e dá outras providências. Revogada pela Lei Federal n ${ }^{\circ} 12.853$ de 14 de agosto de 2013.

BRASIL. Ministério da Educação e Conselho Nacional de Educação. Diretrizes Curriculares Nacionais da Educação Nacional. 2013. Acessado em abril de 2016: http://portal.mec.gov.br/index.php?option=com_docman\& view $=$ download \&alias $=13677$-diretrizes-educacao-basica2013-pdf\&Itemid=30192.

BRASIL. Resolução CNE/CES 4 - Conselho Nacional de Educação / Câmara de Educação Superior, de 19 de Fevereiro de 2002. Institui Diretrizes Curriculares Nacionais do Curso de Graduação em Fisioterapia.

CALS, J. L.; KOTZ, D. Effective writing and publishing scientific papers, part VI: discussion. J Clin Epidemiol, v.66, n.10, p.1064, 2013c.

CALS, J. W.; KOTZ, D. Effective writing and publishing scientific papers, part III: introduction. J Clin Epidemiol, v.66, n.7, p.702, 2013 a.

CALS, J. W.; KOTZ, D. Effective writing and publishing scientific papers, part VIII: references. J Clin Epidemiol, v.66, n.11, p.1198, $2013 b$.

CARTER, M.; FERZLI, M.; WIEBE, E. N. Writing to learn by learning to write in the disciplines. Journal of Business and Technical Communication, v.21, n.3, p.278302, 2007.

CARVAlHO, M. S.; TRAVASSOS, C.; COELI, C.M. Contra a cultura do corta \& cola. Cad. Saúde Pública, v.30, n.5, p.905-907, 2014.
CHUR-HANSEN, A. Medical students' essay-writing skills: criteria-based self and tutor-evaluation and the role of language background. Med Educ, v.34, p.194-198, 2000.

CHUR-HANSEN, A.; VERNON-ROBERTS, J. The evaluation of undergraduate students' written English language skills. Med Educ, v.34, p.642-647, 2000.

COLE, A. F. Plagiarism in graduate medical education. Fam Med, v.39, n.6, p.436-438, 2007.

DE VOSS, D.; ROSATI, A.C. "It wasn't me, was it?" Plagiarism and the web. Computers and Composition, v.19, p.191-203, 2002.

GHAJARZADEH, M.; HASSANPOUR, K.; FERESHTEHNEJAD, S. M.; JAMALI, A.; NEDJAT, S.; ARAMESH, K. Attitude towards plagiarism among Iranian medical students. J Med Ethics, v.39, n.4, p.249, 2013.

GHAJARZADEH, M.; NOROUZI-JAVIDAN, A.; HASSANPOUR, K.; ARAMESH, K.; EMAMI-RAZAVI, S. H. Attitude toward plagiarism among Iranian medical faculty members. Acta Med Iran, v.50, n.11, p.778-781, 2012.

HARRIS, R. A. The plagiarism handbook. Los Angeles: Pyrczak Publishing, 2001.

HRABAK, M.; VUJAKLIJA, A.; VODOPIVEC, I. HREN, D.; MARUSIC, M.; MARUSIC, A. Academic misconduct among medical students in a post-communist country. Med Educ, v.38, p.276-285, 2004.

INAF - Indicador de Alfabetismo Funcional. Ação Educativa, Instituto Paulo Montenegro e IBOPE Inteligência, 2011.

KIM, K. J.; HWANG, J. Y.; LEE, D. W.; SHIM, M. S. Medical student plagiarism in problem-based learning courses. Letter to the editor. Med Educ Online, v.21, p.30537 eCollection, 2016.

KOTZ, D.; CALS, J. W. Effective writing and publishing scientific papers, part IV: methods. J Clin Epidemiol, v.66, n.8, p.817, 2013.

MARTIN, B. Plagiarism by university students: the problem and some proposals. 1992. Acessado em abril de 2016:

http://www.uow.edu.au/arts/sts/bmartin/pubs/92tert.html.

MAVRINAC, M.; BRUMINI, G.; BILIĆ-ZULLE, L.; PETROVECKI, M. Construction and validation of attitudes toward plagiarism questionnaire. Croat Med J, v.60, n.4, p.269-273, 2010.

MOSKOVITZ, C.; KELLOGG, D. Inquiry-based writing in the laboratory course. Science, v.332, p.919-920, 2011. 
ORLANDI, E.; GUIMARÃES, E. Texto, leitura e redação. Vol III. São Paulo. Secretaria da Educação. Coordenadoria de Estudos e Normas Pedagógicas, 1985.

PEZZIN, M. Z. Metodologia estatística computacional de detecção automatizada do plágio autoral - Uma proposta de interpretação dos resultados do programa Farejador de Plágio. Acessado em agosto de 2015: http://www.plagiarismcombat.com/metodologia_do_fareja dor.pdf.

RATHORE, F. A.; WAQAS, A.; ZIA, A. M.; MAVRINAC, M.; FAROOQ F. Exploring the attitudes of medical faculty members and students in Pakistan towards plagiarism: a cross sectional survey. PeerJ, v.18, n.3, p.e1031, 2015.

REYNOLDS, J. A.; THAISS, C.; KATKIN, W.; THOMPSON-JR, R. J. Writing-to-learn in undergraduate science education: a community-based, conceptually driven approach. CBE Life Sci Educ, v.11, p.17-25, 2012.

REYNOLDS, J. A.; THOMPSON-JR, R. J. Want to improve undergraduate thesiswriting? Engage students and their faculty readers in scientific peer review. CBE Life Sci Educ, v.10, n.2, p.209-215, 2011.

ROCHA, V. M.; CALDAS, M. A. J.; ARAÚJO, F. R. O.; RAGASSON, C. A. P.; SANTOS, M. L. M.; BATISTON, A. P. As diretrizes curriculares e as mudanças na formação de profissionais fisioterapeutas (ABENFISIO). Fisioterapia Brasil, p.11-15, 2010.

SENGUPTA, S.; SHUKLA, D.; RAMULU, P.; NATARAJAN, S.; BISWAS, J. Publish or perish: The art of scientific writing. Indian J Ophthalmol, v.62, n.11, p.1089-1093, 2014.

SHIRAZI, B.; JAFAREY, A. M.; MOAZAM, F. Plagiarism and the medical fraternity: a study of knowledge and attitudes. J Pak Med Assoc, v.60, n.4, p.269-273, 2010.

STANLICK, N. An open letter to faculty: some thoughts on plagiarism from "colonel cheatbuster". Faculty Focus. 2008; 7(4). Acessado em abril de 2016 : <http://www.fctl.ucf.edu/Publications/FacultyFocus/conte nt/2008/october.pdf>.

WEEK, A. D. Google could be the way forward. BMJ.; Comment on: Role of systematic reviews in detecting plagiarism: case of Asim Kurjak. 333: 706. 2006 